OPEN ACCESS

*Correspondence:

Martin Tiefenthaler martin.tiefenthaler@i-med.ac.at

tORCID:

Markus Pirklbauer orcid.org/0000-0002-1211-0253

Martin Tiefenthaler orcid.org/0000-0002-5905-7955

Received: 29 September 2021 Accepted: 21 December 2021

Published: 13 January 2022

Citation:

Pirklbauer M, Berger M, Boban MD and Tiefenthaler $M$ (2022) The Tangential Extraperitoneal Retrorenal Approach in Kidney Transplant Biopsy: An Observational Study to Assess Complication and Adequacy Rates. Transpl Int 35:10068. doi: 10.3389/ti.2021.10068
The Tangential Extraperitoneal Retrorenal Approach in Kidney Transplant Biopsy: An Observational Study to Assess Complication and Adequacy Rates

\author{
Markus Pirklbauer ${ }^{\dagger}$, Martin Berger, Miro D. Boban and Martin Tiefenthaler ${ }^{* t}$ \\ Department of Internal Medicine IV - Nephrology and Hypertension, Medical University Innsbruck, Innsbruck, Austria
}

Introduction: Ultrasound-guided percutaneous kidney allograft biopsy is the goldstandard for pathology work-up. Recent studies postulate better safety and efficacy for tangential approaches, however, there is no recommendation regarding biopsy needle path. In this context, we previously described the unified tangential extraperitoneal retrorenal (TER) approach for standard allograft biopsy.

Methods: A single-center retrospective observational study evaluated safety and efficacy of the TER biopsy approach among 250 patients that underwent 330 ultrasound-guided kidney transplant biopsies between January 2011 and May 2020.

Results: The overall major complication rate was $0.56 \%$ per biopsy attempt (1.21\% per biopsy) including blood transfusion, arterial embolization and bladder catheterization for gross hematuria in $0.28,0.14$ and $0.14 \%$ of biopsy attempts, respectively $(0.61,0.30$ and $0.30 \%$ of biopsies, respectively). Minor complications included subcapsular and/or perinephric hematoma, superficial bleeding, arteriovenous fistula and gross hematuria in $12.6,3.0,2.5$ and $1.4 \%$ of biopsy attempts, respectively $(27.0,6.4,5.5$ and $3.0 \%$ of biopsies, respectively). Sample adequacy rate was $86.7 \%$, ranging from 82.2 to $94.1 \%$ if one or $\geq$ two cores were analyzed, respectively. Residents and consultants yielded similar complication and adequacy rates.

Conclusion: According to current literature, ultrasound-guided TER kidney transplant biopsy is a safe and efficient approach eligible for nephrology training.

Keywords: percutaneous kidney transplant biopsy, tangential extraperitoneal retrorenal approach, ultrasoundguided biopsy, complication rate, adequacy rate

Abbreviations: AVF, arteriovenous fistulas; CT, computer tomography; G, gauge; TER, tangential extraperitoneal retrorenal. 


\section{The tangential extraperitoneal retrorenal (TER) approach in kidney transplant biopsy: An observational study to assess complication and adequacy rates}

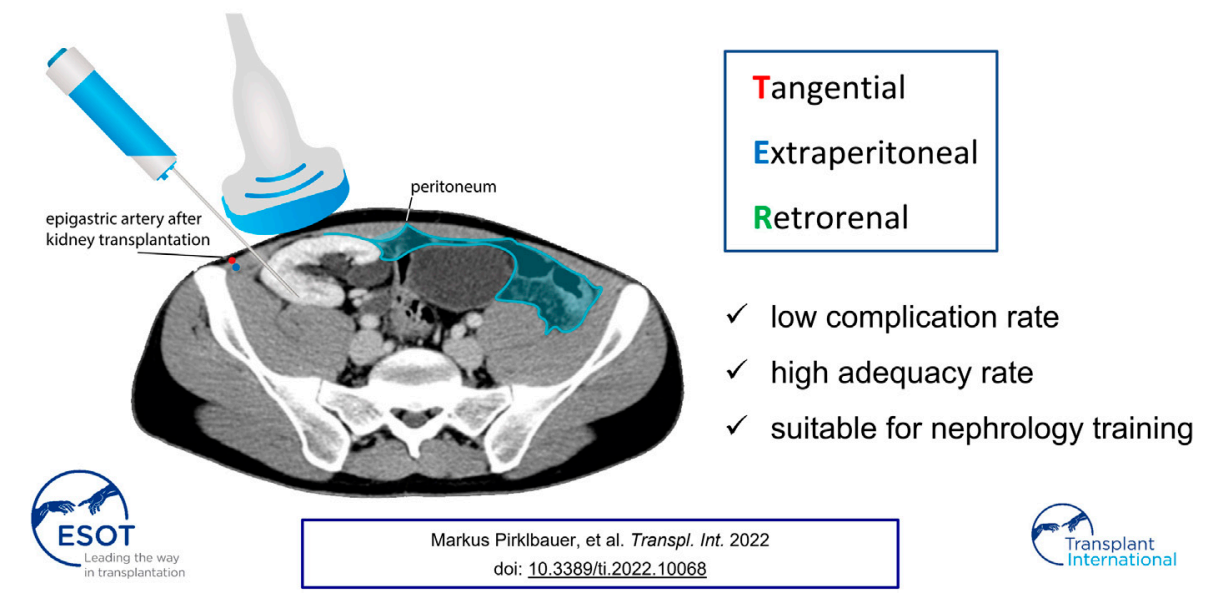

\section{INTRODUCTION}

Ultrasound-guided percutaneous renal transplant biopsy is the gold-standard procedure for allograft pathology work-up. Recent studies, including previous research at our institution, postulate better safety and efficacy of tangential compared to radial approaches [1-3], however, there is no general consensus regarding biopsy needle path for this standard technique. A tangential biopsy allows to direct the needle tip away from the renal hilum, the ureter, and large vessels of the anastomosis region, thereby sparing these anatomical structures from potential injury. In this regard, we recently developed the so called tangential, extraperitoneal, retrorenal (TER) approach for standard allograft biopsy, that penetrates the allograft parallel to the renal capsule (tangential component, T), keeps safe distance to the peritoneal fold (extraperitoneal component, E), and targets the posterior side of the allograft (retrorenal component, $\mathrm{R}$ ) in a lateral-to-medial approach. A pilot study among 104 patients already demonstrated excellent safety and efficacy of the TER approach in 127 kidney transplant biopsies [1]. In our present study we verify these results in a larger patient cohort by demonstrating excellent complication and adequacy rates among 250 patients undergoing 330 kidney transplant biopsies utilizing a conventional (96.1\%) or modified (3.9\%) TER approach. Furthermore, this is the first study to 1) assess both major and minor complications based on a standardized postprocedural ultrasound follow-up as well as to 2) confirm the eligibility of TER kidney transplant biopsy for nephrology training.

\section{PATIENTS AND METHODS}

A single-center retrospective observational study was conducted at our Department to assess safety and efficacy of TER kidney transplant biopsy. Between January 2011 and May 2020, 250 patients underwent at least one kidney transplant biopsy at our institution and were included in the present study. The TER approach is the standard technique for kidney transplant biopsy at our institution and was performed in 317/330 allograft biopsies (96.1\%). A modified TER approach, which featured only two of the three components of the conventional TER approach (tangential, extraperitoneal, retrorenal), had to be conducted in 13/330 biopsies (3.9\%) due to anatomical causes, e.g., dislocated inferior epigastric artery, orthotopic allograft transplantation or preexisting hematoma. 104 of 250 study patients were already included in our previous pilot study [1]. 6/330 allograft biopsies (1.8\%) were protocol biopsies, the remaining 324 biopsies were based on indication. Patient data were available from the institutions' computerized clinical documentation systems.

\section{Biopsy Protocol}

Kidney transplant biopsy was exclusively performed in an inpatient setting where patients are admitted to hospital on the day of biopsy and discharged on the following day. Antiplatelet/anti-coagulant medication was halted from 7-14 days prior to 7-14 days after biopsy depending on the type of drug. Patients at high risk of thromboembolism were administered enoxaparin-sodium during that period; however, enoxaparinsodium was administered no later than $24 \mathrm{~h}$ before biopsy. Blood pressure and heart rate were monitored periinterventionally. Anti-hypertensive medication (e.g., nitroglycerine and/or urapidil and/or dihydralazine) was administered if blood pressure peri-interventionally exceeded $160 / 90 \mathrm{mmHg}$. Lorazepam sedation was available for episodes of anxiety and/or agitation; however, patients did not receive general anesthesia. Following biopsy, patients had to remain in a supine position and use an abdominal belt to minimize the risk of hematoma. Monitoring ended $5 \mathrm{~h}$ after biopsy if a post-biopsy urine void and no signs of gross hematuria, flank pain or other symptoms indicating a complication were reported. A blood count 


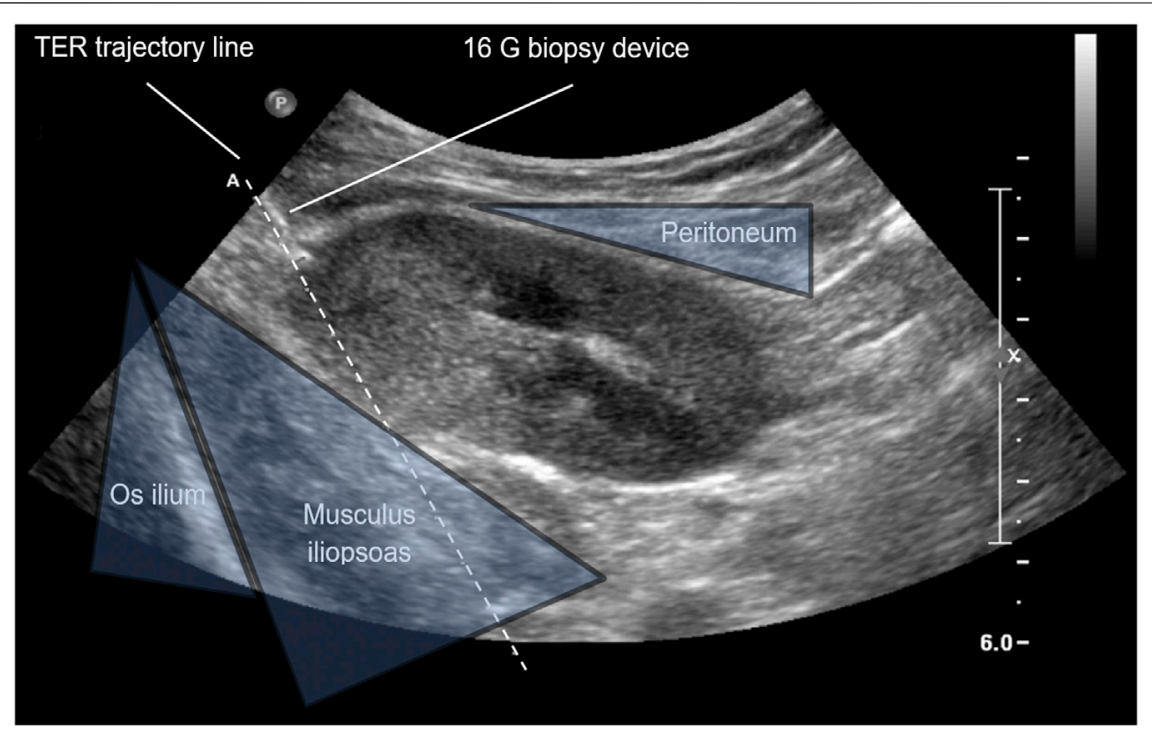

FIGURE 1 | Anatomic landmarks of real-time ultrasound-guided kidney allograft biopsy. Ultrasound image of the right iliac kidney allograft; TER, tangential, extraperitoneal, retrorenal; G, gauge.

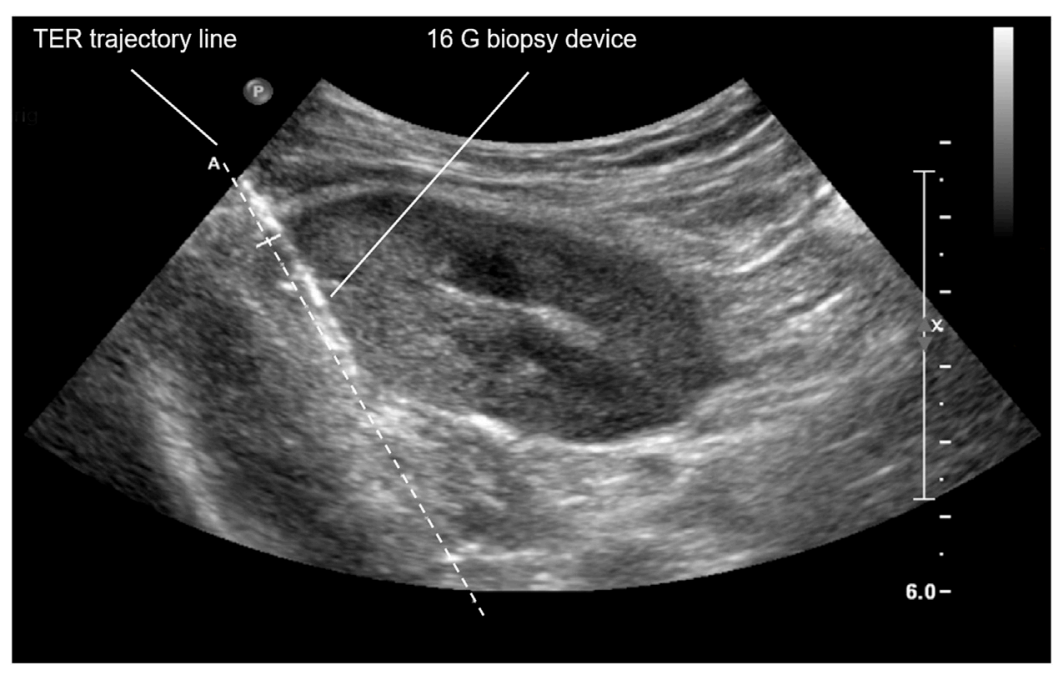

FIGURE 2 | TER biopsy of right iliac kidney allograft. TER, tangential, extraperitoneal, retrorenal; G, gauge.

as well as color-duplex ultrasound examination was performed on the next day to detect bleeding complications or arterio-venous fistulas (AVF). If both examinations yielded normal findings, patients were discharged from hospital and instructed to avoid weight-lifting $>5 \mathrm{~kg}$ and contact sports for 14 days. Normal physical activity including running and cycling was encouraged to prevent thromboembolism. Patients were asked to immediately return to the hospital in case of discomfort after discharge.

\section{Biopsy Technique}

Real-time ultrasound-guided TER kidney transplant biopsy is performed in supine or-in case of obesity or pendulous abdomen-lateral decubitus position. A needle guidance system mounted to the ultrasound transducer optimizes needle handling and helps to visualize needle path. The ultrasound transducer is placed approximately $2 \mathrm{~cm}$ medial to the anterior superior iliac spine to determine optimal biopsy area. The latter allows to 1) penetrate the allograft parallel to the renal capsule (i.e., tangential component), 2) keep safe distance to the peritoneal fold (extraperitoneal component), and 3) target the posterior side of the upper pole or the most dorsal part of the lateral portion of the allograft (retrorenal component) (Figure 1). Local anesthesia with xylocaine $2 \%$ is administered as subcutaneous depot prior to skin incision as well as ultrasound-guided deep depot along the needle path up to the renal capsule. Via a small skin incision the biopsy device is then advanced towards the allograft from lateral to medial in a transverse 
TABLE 1 | Patient characteristics.

\begin{tabular}{lc}
\hline Patients & 250 \\
Male & $155(62.0)$ \\
Female & $95(38.0)$ \\
No. of performed biopsies & 330 \\
On male patients & $203(61.5)$ \\
On female patients & $127(38.5)$ \\
Per patient & \\
1 & $194(77.6)$ \\
2 & $39(15.6)$ \\
3 & $11(4.4)$ \\
4 & $5(2.0)$ \\
5 & $1(0.4)$ \\
No. of kidney transplant & $1.41 \pm 0.8(1-7)$ \\
Age (years) & $50(18-78)$ \\
Body Mass Index (kg/m ${ }^{2}$ ) & $24.3(15.6-42.0)$ \\
Arterial hypertension $(\geq 140 / 90 \mathrm{mmHg})$ & $291(88.2)$ \\
Diabetes mellitus & $76(23.0)$ \\
Arterial hypertension and diabetes mellitus & $69(20.9)$
\end{tabular}

Data are presented as numbers (percent), mean \pm standard deviation (range), or median (range) for age and body mass index.

No., number.

\begin{tabular}{lc}
\hline TABLE 2 | Biopsy characteristics. \\
\hline No. of performed biopsies & \\
No. of biopsy attempts per biopsy & \\
1 & $28(8.5)$ \\
2 & $237(71.8)$ \\
3 & $57(17.3)$ \\
4 & $4(1.2)$ \\
5 & $4(1.2)$ \\
No. of biopsy attempts & $2.2 \pm 0.6(1-5)$ \\
Total & 709 \\
No. of samples recovered & $1.9 \pm 0.5(1-5)$ \\
Total & $637(89.9)$ \\
No. of biopsy attempts without recovery of sample & $72(10.2)$ \\
Biopsy technique & \\
No. of performed biopsies & 330 \\
Using TER approach & $317(96.1)$ \\
Using modified TER approach & $13(3.9)$ \\
No. of biopsy attempts & 709 \\
Using TER approach & $683(96.3)$ \\
Using modified TER approach & $26(3.7)$ \\
\end{tabular}

Data are displayed as number (percent) or mean \pm standard deviation (range). ND, no data; No., number.

plane using real-time ultrasound guidance. Once the renal capsule is reached, the biopsy needle is fired tangentially into the outer third of the renal cortex (Figure 2). Bedside analysis of biopsy cores for adequacy was routinely done by using a magnifying glass for the crude assessment of glomerular number. Whenever feasible, at least two core samples measuring $1.3 \mathrm{~mm}$ in diameter and $22 \mathrm{~mm}$ in length are obtained using a $16 \mathrm{~cm}$ long, 16 Gauge $(G)$ spring-loaded biopsy device (Bard ${ }^{\circledast}$ Monopty ${ }^{\circledR}$ Disposable Core Biopsy Instrument).

\section{Definition of Complications}

Major complications were defined as biopsy-related complications requiring invasive therapy and included bladder catheterization for gross hematuria, blood transfusion (following either a biopsy-related drop of hemoglobin or image confirmation of biopsy-related bleeding), interventional radiology procedure with or without arterial embolization, surgery, graft loss, or death. Minor complications were defined as any biopsy-related relevant medical condition not requiring invasive therapy. Complication rates were calculated per biopsy attempt and biopsy event.

\section{Definition of Adequacy}

According to the criteria of the Banff 97 working classification of renal allograft pathology [4], a biopsy core sample was considered 1) adequate if it contained at least 10 glomeruli and two arteries or 2) minimal if it contained a minimum of seven glomeruli and one artery in the pathologist's assessment. Adequacy rates given in the present study represent the sum of samples deemed either minimal or adequate. Adequacy was calculated per biopsy as glomerular and arterial yield were reported per biopsy only.

\section{Statistical Analysis}

Descriptive statistics was performed using Microsoft Excel (Microsoft Corporation, Redmond, Washington, United States). Results and baseline characteristics are presented as absolute frequencies or mean values \pm standard deviation (range). $\mathrm{Chi}^{2}$ statistics was performed with SPSS version 24.0 to assess potential associations between nominal parameters (i.e., training status, occurrence of complications and sample adequacy). The level of significance ( $p$ value) was set to 0.05 .

\section{Statement of Ethics}

The study was conducted in accordance with the World Medical Association Declaration of Helsinki. The study protocol was reviewed and approved by the Innsbruck Medical University ethics committee prior to study initiation (approval number ECS 1106/2020). Patient information was managed entirely coded. All patient associated data are subject to privacy protection according to the current European General Data Protection Regulation. Based on the retrospective study design the Innsbruck Medical University ethics committee granted an exemption from requiring written informed consent.

\section{RESULTS}

\section{Patient and Biopsy Characteristics}

330 ultrasound-guided kidney transplant biopsies were performed among 250 patients between January 2011 and May 2020. 203 (61.5\%) and 127 (38.5\%) biopsies were performed on male and female patients, respectively. 194 (77.6\%) patients underwent one biopsy, however, patients were subjected to kidney transplant biopsy up to five times. 2, 3, 4 and 5 biopsies were performed in 39 (15.6\%), 11 (4.4\%), 5 (2.0\%) and $1(0.4 \%)$ patient, respectively. Median age and body mass index at the time of biopsy was 50 years (range 18-78) and 24.3 (range 15.6-42.0), respectively. The mean number of kidney transplants per patient was $1.41 \pm 0.8$ (range $1-7)$. The total 
TABLE 3 | Biopsy complications.

\begin{tabular}{|c|c|c|c|c|}
\hline & \multicolumn{2}{|c|}{ TER + modified TER } & \multicolumn{2}{|c|}{ TER only } \\
\hline & 709 biopsy attempts & 330 biopsies & 683 biopsy attempts & 317 biopsies \\
\hline \multicolumn{5}{|l|}{ Overall } \\
\hline Minor & $149(21.0)$ & $149(45.2)$ & $141(20.6)$ & $141(44.5)$ \\
\hline Major & $4(0.6)$ & $4(1.2)$ & $2(0.3)$ & $2(0.6)$ \\
\hline Total & $153(21.6)$ & $153(46.4)$ & $143(20.9)$ & $143(45.1)$ \\
\hline \multicolumn{5}{|l|}{ Periprocedural minor complications } \\
\hline Drainage of serous fluid & $2(0.3)$ & $2(0.6)$ & $2(0.3)$ & $2(0.6)$ \\
\hline Vasovagal reaction & $5(0.7)$ & $5(1.5)$ & $5(0.7)$ & $5(1.6)$ \\
\hline Hypertensive urgency & $1(0.1)$ & $1(0.3)$ & $1(0.2)$ & $1(0.3)$ \\
\hline Superficial bleeding & $21(3.0)$ & $21(6.4)$ & $19(2.8)$ & $19(6.0)$ \\
\hline \multicolumn{5}{|l|}{ Postprocedural minor complications } \\
\hline Gross hematuria & $10(1.4)$ & $10(3.0)$ & $10(1.5)$ & $10(3.2)$ \\
\hline Arteriovenous fistula & $18(2.5)$ & $18(5.5)$ & $18(2.6)$ & $18(5.7)$ \\
\hline Subcapsular hematoma & $7(1.0)$ & $7(2.1)$ & $6(0.9)$ & $6(1.9)$ \\
\hline Perinephric hematoma & $82(11.6)$ & $82(24.9)$ & $78(11.4)$ & $78(24.6)$ \\
\hline$<3 \times 1 \mathrm{~cm}$ & $69(9.7)$ & $69(20.9)$ & $66(9.7)$ & $66(20.8)$ \\
\hline$>3 \times 1 \mathrm{~cm}$ & $8(1.1)$ & $8(2.4)$ & $7(1.0)$ & $7(2.2)$ \\
\hline ND & $5(0.7)$ & $5(1.5)$ & $5(0.7)$ & $5(1.6)$ \\
\hline Pain $^{a}$ & $2(0.3)$ & $2(0.6)$ & $1(0.2)$ & $1(0.3)$ \\
\hline Deep vein thrombosis & $1(0.1)$ & $1(0.3)$ & $1(0.2)$ & $1(0.3)$ \\
\hline \multicolumn{5}{|l|}{ Major complications } \\
\hline Rinsing catheter for gross hematuria & $1(0.1)$ & $1(0.3)$ & $1(0.2)$ & $1(0.3)$ \\
\hline Transfusion & $2(0.3)$ & $2(0.6)$ & $1(0.2)$ & $1(0.3)$ \\
\hline Coiling/arterial embolization & $1(0.1)$ & $1(0.3)$ & $0(0.0)$ & $0(0.0)$ \\
\hline
\end{tabular}

${ }^{a}$ No ultrasound correlate.

Data are displayed as number (percent).

ND, no data; No., number.

number of biopsy attempts was 709, yielding 637 core samples. Mean number of biopsy attempts per biopsy and recovered core samples was $2.2 \pm 0.6$ (range 1-5) and $1.9 \pm 0.5$ (range 1-5), respectively. TER biopsy was performed in $317 / 330$ biopsies (96.1\%) and 683/709 biopsy attempts (96.3\%). In 13 biopsies (3.9\%) and 26 biopsy attempts (3.7\%), a modified TER approach had to be applied due to anatomical causes, e.g., dislocated inferior epigastric artery, orthotopic allograft transplantation or preexisting hematoma. Tangential, extraperitoneal or retrorenal biopsy could not be performed in four, two and seven biopsies, respectively. Though, at least two components of the conventional TER approach were performed in these 13 cases (Tables 1, 2).

\section{Major Complications}

Among 709 biopsy attempts (330 biopsies), four major complications $(0.6 \%$ of biopsy attempts and $1.2 \%$ of biopsies) were documented among 3 patients throughout the study period. Considering conventional TER approach only with 683 biopsy attempts ( 317 biopsies), 2 complications ( $0.3 \%$ of biopsy attempts and $0.6 \%$ of biopsies) were classified as major complications. One patient was subject to rinsing catheterization of the bladder $(0.1 \%$ of biopsy attempts and $0.3 \%$ of biopsies) due to gross hematuria following biopsy. Transfusion of blood products ( $0.3 \%$ of biopsy attempts and $0.6 \%$ of biopsies) was required in two patients. One of these patients underwent conventional TER kidney transplant biopsy and received two units of packed red blood cells on the day after biopsy on account of suspected bleeding in abdominal ultrasound examination and CT scan. The other patient underwent a modified TER approach (radial biopsy) and
TABLE 4 | Sample adequacy.

No. of performed biopsies

No. of analyzed samples

0

$1 \quad 174(52.7)$

$2+150(45.5)$

ND

$2(0.6)$

$2(0.6)$

No. of biopsies considered

Adequate

$192(58.2)$

Minimal

94 (28.5)

Inadequate

ND

Adequate and minimal

If 1 sample analyzed

If 2 samples analyzed

If 3 samples analyzed

If 2 or 3 samples analyzed

$42(12.7)$

$2(0.6)$

286 (86.7)

143 (82.2)

$141(94.0)$

2 (100.0)

$143(94.1)$

No. of glomeruli

$15.7 \pm 9.3(0-69)$

No. of arteries

$2.5 \pm 1.6(0-10)$

Data are displayed as number (percent) or mean \pm standard deviation (range). ND, no data; No., number.

experienced aggravated pain immediately after biopsy. Instant ultrasound examination revealed arterial bleeding involving the upper pole renal artery. Emergency coiling $(0.1 \%$ of biopsy attempts and $0.3 \%$ of biopsies) was conducted and four units of packed red blood cells and platelet concentrates were administered for low hemoglobin and platelet count. No patient required surgical treatment. No graft losses or deaths occurred (Table 3 ). 


\section{Minor Complications}

Overall, 149 events were classified as minor complications $(21.0 \%$ of biopsy attempts and $45.2 \%$ of biopsies). Considering TER approach only, 141 minor complications were documented (20.6\% of biopsy attempts and $44.5 \%$ of biopsies). Routine ultrasound examination on day 1 after kidney transplant biopsy identified 82 perinephric hematomas, eighteen AVF, and seven subcapsular hematomas (i.e., 11.6, 2.5 and 1.0\% of biopsy attempts, respectively and 24.9, 5.5 and $2.1 \%$ of biopsies, respectively). Of the perinephric hematomas, 69 were smaller than $3 \times 1 \mathrm{~cm}$, eight were bigger than $3 \times 1 \mathrm{~cm}$ and five could not be categorized because of missing data (i.e., 9.7, 1.1 and $0.7 \%$ of biopsy attempts, respectively and 20.9, 2.4 and 1.5\% of biopsies, respectively). All AVF had resolved spontaneously at follow-up ultrasound examination. 21 superficial bleedings, 10 episodes of gross hematuria, 5 vasovagal reactions requiring atropine administration, and one hypertensive urgency requiring administration of urapidil, dihydralazine and amlodipine, were detected after kidney allograft biopsy (i.e., 3.0, 1.4, 0.7, and $0.1 \%$ of biopsy attempts, respectively and $6.4,3.0,1.5$ and $0.3 \%$ of biopsies, respectively). Abdominal pain ( $0.3 \%$ of biopsy attempts and $0.6 \%$ of biopsies) was reported in two patients. In both cases, no ultrasound correlate was detected and both patients were administered analgesic medication. One case of deep vein thrombosis $(0.1 \%$ of biopsy attempts and $0.3 \%$ of biopsies $)$ of the ipsilateral popliteal vein was documented (Table 3).

\section{Sample Adequacy}

Cores samples were evaluated according to the Banff 97 working classification of renal allograft pathology [4]. 192 (58.2\%) and 94 $(28.5 \%)$ biopsies were considered adequate and minimal, respectively. Thus, a total of 286 biopsies (86.7\%) met the criteria for sample adequacy. 42 biopsies (12.7\%) were considered inadequate and data from two biopsies $(0.6 \%)$ were lacking. Adequacy rate increased to $94.1 \%$, if two or more core samples were analyzed. Mean number of glomeruli and arteries was $15.7 \pm 9.3$ glomeruli (range $0-69$ ) and $2.5 \pm 1.6$ arteries (range $0-10$ ), respectively (Table 4 ).

\section{Complications and Sample Adequacy of Training Biopsies}

$116(35.2 \%)$ and $214(64.9 \%)$ biopsies were performed by nephrology residents and consultants, respectively. Major and minor complications occurred in 1.2 and $20.5 \%$ of resident biopsy attempts, respectively (2.6 and $44.0 \%$ of resident biopsies, respectively) and 0.2 and $21.3 \%$ of consultant biopsy attempts, respectively ( 0.5 and $45.8 \%$ of consultant biopsies, respectively). $p=0.094$ and $p=0.798$ for association of resident status with major and minor complications, respectively. Considering TER approach only, major complication rate per biopsy attempt was 0.4 and $0.2 \%$ among 249 (35.1\%) resident and $460(64.9 \%)$ consultant biopsy attempts, respectively (i.e., 0.9 and $0.5 \%$ among 111 resident and 206 consultant biopsies, respectively). Overall adequacy rate was 87.9 and $86 \%$ for biopsies performed by residents and consultants, respectively
( $p=0.619$ for association of resident status with sample adequacy) (Supplementary Tables S1, S2).

\section{DISCUSSION}

The present study reinforces the results of a recent pilot study [1] demonstrating excellent safety and efficacy of TER kidney transplant biopsy and corroborates previous findings showing low major complication and high adequacy rates with the use of tangential kidney allograft biopsy [2, 3, 5]. With a major complication rate of $0.3 \%$ per biopsy attempt $(0.6 \%$ per biopsy) the TER approach is among the safest allograft biopsy approaches according to current literature (Supplementary Table S3). Major complication rates have been previously demonstrated to be up to $5.6 \%$ [6]; however the latter study did not report a specific biopsy region or needle path. Comparable studies utilizing a tangential biopsy technique reported major complication rates ranging between $0.0 \%$ [5] and $3.6 \%$ [7]. While the former study first described the so called "cortex-only" view among 188 biopsies, the latter study used a computer tomography (CT)-guided approach among 28 biopsies. While small patient number is a substantial limitation of both studies, CT-guided approaches implicate additional risk from radiation exposure. The most comprehensive studies assessing ultrasound-guided tangential allograft biopsy yielded major complication rates of 0.7 [2], 0.3 [3] and 1.9\% [8]. Minor complications, such as AVF and hematomas, are best detected through standardized post-procedural ultrasound examination and/or blood count; however, most of the available studies, including comparable studies with tangential biopsy techniques $[2,3,5,8]$, did not routinely perform postprocedural ultrasound and/or blood count. Thus, it is likely to speculate that these studies might not reflect the true incidence of minor complications. By performing routine ultrasound and blood count on the day after biopsy, our study is the first to comprehensively assess both symptomatic and asymptomatic complications. Based on these substantial differences in postprocedural management, however, the minor complication rates found in the present study are not comparable to previous studies in the field. AVF are usually asymptomatic and rarely require specific therapy; however, centers performing ultrasound-based screening report AVF rates of up to $10.7 \%$ [9]. Generally, AVF rate seems to correlate with both the extent and timing of postprocedural ultrasound examination. Consequently, AVF rates are usually reported to be low in studies that do not routinely perform post-procedural imaging [7,10-13] and tend to be higher in studies that perform ultrasound examination within hours $[9,14$, 15 ] as compared to those performing immediate post-procedural ultrasound [16-18].

The low AVF rate (2.5\% per biopsy attempt and 5.5\% per biopsy) found with the TER biopsy approach is likely to result from targeting the outer third of cortical renal parenchyma and thus, from sparing larger vessels in the medullary region. All AVF spontaneously resolved at a 2 weeks follow-up examination. Nevertheless, screening might be beneficial for individual patients as AVF-associated severe complications, such as 


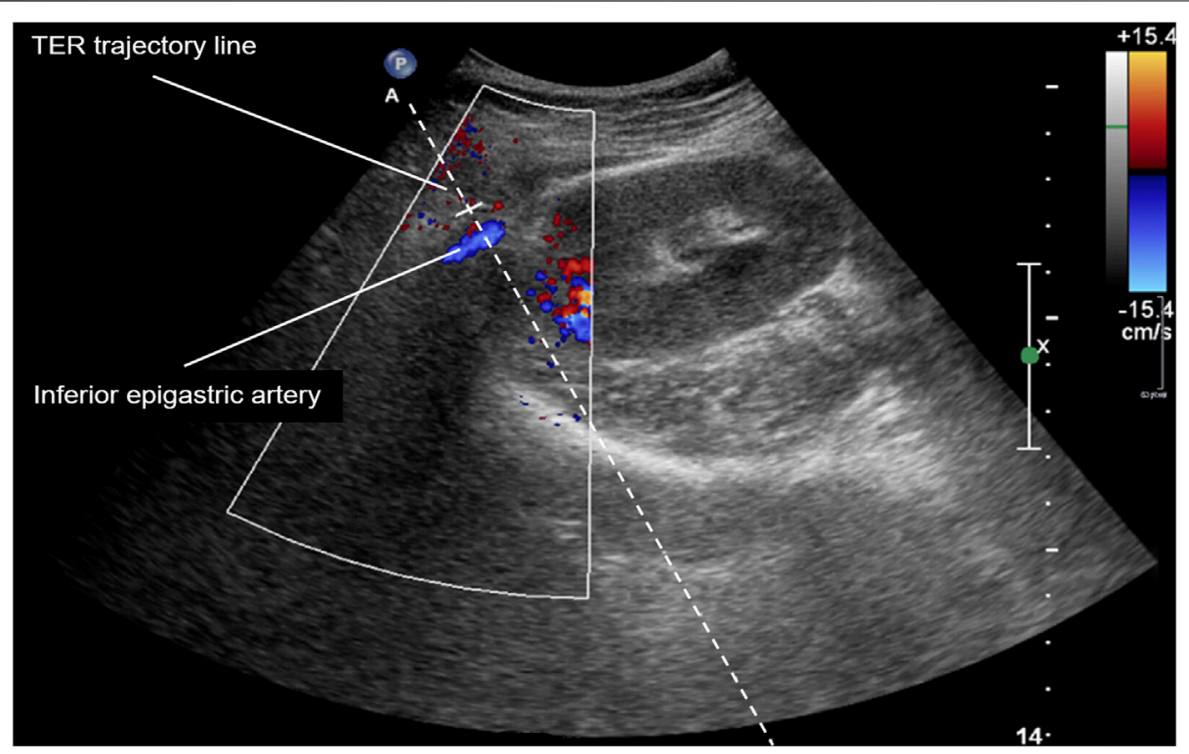

FIGURE 3 | Color-duplex ultrasound image of right iliac kidney allograft and dislocated inferior epigastric artery. TER, tangential, extraperitoneal, retrorenal.

arterial embolization and nephrectomy have been described in the literature [10, 15]. A recent retrospective Japanese study described an AVF rate of $2.6 \%$ after kidney allograft biopsy and proposed that embolization is a safe treatment for these AVF. However, the authors state that the study was likely to underestimate AVF rate as post-procedural management was not consistent among the study population [19].

As for AVF, hematoma detection rate correlates with the availability of post-procedural ultrasound examination and ranges from $0.0 \%$ in studies that did not perform ultrasound examination [20] and $13.4 \%$ [21] in studies that performed immediate postprocedural ultrasound. With a consequent post-procedural ultrasound examination, the present study reports an overall hematoma rate of $12.6 \%$ per biopsy attempt (27.0\% per biopsy). A previous study performing comparable post-procedural management reported a similar hematoma rate of $11.1 \%$ [22]. While none of the reported perinephric $(11.6 \%$ of biopsy attempt and $24.9 \%$ of biopsies) and subcapsular (1.0\% of biopsy attempts and $2.1 \%$ of biopsies) hematomas required specific therapy in our study, individual patients might benefit from hematoma screening as large hematomas may profit from extended period of rest or-if applicable-extended period of anti-platelet/anticoagulant withdrawal post biopsy. Additionally, post-procedural screening might help to timely identify large hematomas that will require surgical evacuation in order to preserve kidney allograft function [6, 8, 13]. Nevertheless, substantial heterogeneity exists in post-procedural management of kidney transplant biopsy between facilities, partly due to reimbursement issues. While overnight inhospital observation is clinical routine in many European and Japanese centers, others, including most U.S. facilities, perform shorter observation periods. In this regard, a recent study by Patel et al. both corroborated the low rate of major bleeding complications with ultrasound-guided renal transplant biopsy
(0.2\%) and presented evidence that a standardized 1-hour postprocedure observation protocol can be safely used. However, the authors state that more than half of these complications were not clinically apparent within $4 \mathrm{~h}$ of biopsy [23]. Overnight in-hospital observation is part of the routine post-procedural management at our facility, however, the present study does not advocate any specific post-procedural management strategy at this time.

The occurrence of gross hematuria following kidney allograft biopsy ranges from $0.0 \%$ [24] and 9.0\% [13] in the literature. Our finding of a rather low gross hematuria rate (1.4\% per biopsy attempt and $3.0 \%$ per biopsy) is consistent with the low rate $(0.7 \%)$ found in another tangential allograft biopsy study [2]. Other minor complications, such as superficial bleedings, vasovagal reactions, hypertensive urgency, seroma drainage, and aggravated pain, are rarely reported in the literature, and thus, occurrence rates are difficult to compare. Deep vein thrombosis that is normally associated with immobilization might not be considered as direct biopsy complication. Previous studies reported that renal allograft biopsy within 30 days after transplantation, deep puncture (i.e., high percentage of medulla) and the number of biopsy attemps per biopsy increase the risk of AVF $[25,26]$. In our study, 47/330 biopsies (14\%) were performed within 30 days after kidney transplantation, however, we did not find a significant association with major and/or minor complications. Interestingly, the latter did also not significantly correlate with the number of biopsy attempts per biopsy. While major complications exclusively occurred among patients that were subject to 2 biopsy attempts, AVF rate did not increase with the number of biopsy attempts per biopsy (up to 5). Albeit not statistically significant, hematoma rate nominally increased from $25 \%$ (with up to 4 biopsy attempts) to $50 \%$ (with 5 biopsy attempts $)(p=0.24)$. Data regarding the percentage of medulla 
in biopsy specimen is not available for the present study, however, deep puncture would be a rare finding with adequate TER biopsy as parallel orientation of the biopsy needle to the renal capsule should avoid any deep puncture.

Sample adequacy rates range from 52.9 [20] to $99.5 \%$ [8] in the literature (Supplementary Table S4). However, some of the previous studies $[5,20,27]$ regarded glomerular yield only, and thus, might overestimate adequacy. With an overall sample adequacy rate of $86.7 \%$ our study is among the top 6 studies reporting adequacy according to Banff classification. Adequacy rate increased to $94.1 \%$ in our study, if two or more core samples were analyzed. However, the latter applied to only $46.1 \%$ of biopsies due to frequent electron microscopic work-up of a second core sample. It is likely to speculate that adequacy rates would have exceeded $90 \%$ once these samples were analyzed. Based on this finding we now obtain three core samples in case of planned electron microscopic work-up. Biopsy technique and needle size vary among different studies, however, it has been previously stated that adequacy rates rather correlate with biopsy technique than needle size $[24,28]$ (Supplementary Table S4). As we found no significant difference between resident status and complication as well as adequacy rates (Supplementary Tables S1, S2), the novel TER biopsy approach can be considered appropriate for training biopsies. This is in contrast to a previous study stating a possible association of major complications with lesser operator experience in tangential allograft biopsy [2]. Previous studies evaluating tangential biopsy approaches $[2,3]$ did not stipulate a particular biopsy region or needle paths. However, transperitoneal needle paths are more likely to cause intraperitoneal hematoma and medial-to-lateral biopsy approaches are prone to injure both the rectus sheath and inferior epigastric artery with subsequent development of rectus sheath hematoma. Both complications have been previously reported with tangential biopsy approaches $[2,5]$. With the TER approach these complications are less likely to occur as an exclusive extraperitoneal as well as lateral-to-medial biopsy approach keeps safe distance to both the rectus sheath, inferior epigastric artery, and the peritoneal fold. However, as the inferior epigastric artery might not be located in its usual position along the rectus abdominis muscle but dislocated further lateral due to mobilization during transplantation, the TER biopsy approach should be modified to avoid vascular injury in that case (Figure 3). While tangential cortex biopsy has been previously demonstrated to convey substantial advantages in terms of safety and adequacy $[2,3,5]$, our results do not support the hypothesis that retrorenal biopsy approaches may lead to uncontrollable bleeding [5]. In contrast, the surrounding iliopsoas muscle as well as the dorsal pelvis rather serve as natural barriers against extended hematoma formation. Furthermore, the iliopsoas muscle is the only adjacent organ structure to be accidentally injured in case of a retrorenal biopsy approach. While previous studies inconsistently reported the number of biopsy attempts per biopsy and calculated complication rates per biopsy only, our study is the first to present complication rates per biopsy attempt and biopsy event. The present study is limited by a small sample size.

By demonstrating low major complication $(<1 \%)$ and high sample adequacy rates $(>90 \%$ when two or more samples are analyzed) our present study confirms high safety and efficacy of the novel TER approach for standard kidney transplant biopsy. Furthermore, this is the first study to 1) comprehensively report both major and minor complication rates based on a standardized post-procedural management and 2) confirm the eligibility of the TER approach for supervised training biopsies with respect to safety and efficacy.

\section{CAPSULE SUMMARY SENTENCE}

Ultrasound-guided percutaneous renal transplant biopsy is the gold-standard procedure for allograft pathology work-up. Recent studies, including previous research at our institution, postulate better safety and efficacy of tangential compared to radial approaches, however, there is no general consensus regarding biopsy needle path for this standard technique. In this context, we recently described a unified tangential, extraperitoneal, retrorenal (TER) approach for standard allograft biopsy and demonstrated excellent safety and efficacy in a pilot study (Transpl Int. 2017; 30: 947-50). By penetrating the allograft parallel to the renal capsule (tangential component), keeping safe distance to the peritoneal fold (extraperitoneal component) and targeting the posterior side of the allograft in a lateral-to-medial approach (retrorenal component), the TER approach aims at reducing the risk of intraperitoneal as well as rectus sheet hematoma. By verifying low major complication $(<1 \%)$ and high adequacy $(>90 \%)$ rates among 250 patients undergoing 330 kidney transplant biopsies our present study confirms safety and efficacy of the TER approach for standard ultrasound-guided allograft biopsy. Furthermore, this is the first study to (1) assess both major and minor complications based on a standardized postprocedural ultrasound follow-up as well as to (2) confirm the eligibility of TER kidney transplant biopsy for nephrology training.

\section{DATA AVAILABILITY STATEMENT}

The raw data supporting the conclusions of this article will be made available by the authors, without undue reservation.

\section{ETHICS STATEMENT}

The studies involving human participants were reviewed and approved by Innsbruck Medical University ethics committee, Medical University Innsbruck, Innrain 43, 6020 Innsbruck, Austria; Approval number: ECS 1106/2020. Written informed consent for participation was not required for this study in accordance with the national legislation and the institutional requirements.

\section{AUTHOR CONTRIBUTIONS}

Research idea and study design: MT; data collection: MT, MB, and MDB; data analysis/interpretation: MP, MB, MDB, and MT; statistical analysis: $\mathrm{MB}, \mathrm{MDB}$, and MP; manuscript preparation, drafting, and approval of the final version: MP, MB, MDB, and MT. 


\section{CONFLICT OF INTEREST}

The authors declare that the research was conducted in the absence of any commercial or financial relationships that could be construed as a potential conflict of interest.

\section{REFERENCES}

1. Boban MD, Tiefenthaler M Tangential Extraperitoneal Retrorenal Approach: a Specified Uniform Technique for Renal Transplant Biopsy. Transpl Int (2017) 30:947-50. doi:10.1111/tri.12994

2. Patel MD, Phillips CJ, Young SW, Kriegshauser JS, Chen F, Eversman WG, et al. US-guided Renal Transplant Biopsy: Efficacy of a Cortical Tangential Approach. Radiology (2010) 256:290-6. doi:10.1148/radiol.10091793

3. Patel MD, Young SW, Scott Kriegshauser J, Dahiya N Ultrasound-guided Renal Transplant Biopsy: Practical and Pragmatic Considerations. Abdom Radiol (2018) 43:2597-603. doi:10.1007/s00261-018-1484-5

4. Racusen LC, Solez K, Colvin RB, Bonsib SM, Castro MC, Cavallo T, et al..The Banff 97 Working Classification of Renal Allograft Pathology. Kidney Int (1999) 55:713-23. doi:10.1046/j.1523-1755.1999.00299.x

5. Shin J, Park SY Diagnostic Efficacy and Safety of Ultrasound-Guided Kidney Transplant Biopsy Using Cortex-Only View: a Retrospective Single-center Study. Eur Radiol (2019) 29:5272-9. doi:10.1007/s00330-018-5910-4

6. Tapia-Canelas C, Zometa R, López-Oliva MO, Jiménez C, Rivas B, Escuin F, et al..Complications Associated with Renal Graft Biopsy in Transplant Patients. Nefrologia (2014) 34:115-9. doi:10.3265/Nefrologia.pre2013.Nov.12232

7. Cortesi C, Sedki M, Ruiz P, Salsamendi J, Mattiazzi A Computed TomographyGuided Kidney Transplant Biopsy Outcomes: A Single-Center Experience. Exp Clin Transpl (2020) 18:676-81. doi:10.6002/ect.2019.0111

8. Morgan TA, Chandran S, Burger IM, Zhang CA, Goldstein RB Complications of Ultrasound-Guided Renal Transplant Biopsies. Am J Transpl (2016) 16: 1298-305. doi:10.1111/ajt.13622

9. Furness PN, Philpott CM, Chorbadjian MT, Nicholson ML, Bosmans J-L, Corthouts BL, et al. Protocol Biopsy of the Stable Renal Transplant: a Multicenter Study of Methods and Complication Rates. Transplantation (2003) 76:969-73. doi:10.1097/01.tp.0000082542.99416.11

10. Preda A, Van Dijk LC, Van Oostaijen JA, Pattynama PM Complication Rate and Diagnostic Yield of 515 Consecutive Ultrasound-Guided Biopsies of Renal Allografts and Native Kidneys Using a 14-gauge Biopty Gun. Eur Radiol (2003) 13:527-30. doi:10.1007/s00330-002-1482-3

11. Birk PE, Blydt-Hansen TD, Dart AB, Kaita LM, Proulx C, Taylor G Low Incidence of Adverse Events in Outpatient Pediatric Renal Allograft Biopsies. Pediatr Transpl (2007) 11:196-200. doi:10.1111/j.1399-3046.2006.00659.x

12. Ho QY, Lim CC, Thangaraju S, Siow B, Chin YM, Hao Y, et al. Bleeding Complications and Adverse Events after Desmopressin Acetate for Percutaneous Renal Transplant Biopsy. Ann Acad Med Singap (2020) 49: 52-64. doi:10.47102/annals-acadmedsg.2019164

13. Fang J, Li G, Xu L, Zhang L, Yin W, Lai X, et al. Complications and Clinical Management of Ultrasound-Guided Renal Allograft Biopsies. Transl Androl Urol (2019) 8:292-6. doi:10.21037/tau.2019.07.23

14. Schwarz A, Gwinner W, Hiss M, Radermacher J, Mengel M, Haller H Safety and Adequacy of Renal Transplant Protocol Biopsies. Am J Transpl (2005) 5: 1992-6. doi:10.1111/j.1600-6143.2005.00988.x

15. Franke M, Kramarczyk A, Taylan C, Maintz D, Hoppe B, Koerber F Ultrasound-guided Percutaneous Renal Biopsy in 295 Children and Adolescents: Role of Ultrasound and Analysis of Complications. PLoS One (2014) 9:e114737. doi:10.1371/journal.pone.0114737

\section{SUPPLEMENTARY MATERIAL}

The Supplementary Material for this article can be found online at: https://www.frontierspartnerships.org/articles/10.3389/ti.2021. 10068/full\#supplementary-material

16. Yablon Z, Recupero P, McKenna J, Vella J, Parker MG Kidney Allograft Biopsy: Timing to Complications. Cn (2010) 74:39-45. doi:10.5414/cnp74039

17. Torres-Rodríguez IB, Castella-Fierro E, Serres-Creixans X, Salcedo-Allende M, Azancot-Rivero MA, Perelló-Carrascosa M, et al..Safety and Efficacy of Outpatient Biopsy in Renal Transplantation. Nefrologia (2014) 34:749-55. doi:10.3265/Nefrologia.pre2014.Jul.12509

18. Redfield RR, McCune KR, Rao A, Sadowski E, Hanson M, Kolterman AJ, et al. Nature, Timing, and Severity of Complications from Ultrasound-Guided Percutaneous Renal Transplant Biopsy. Transpl Int (2016) 29:167-72. doi:10.1111/tri.12660

19. Osako K, Yazawa M, Terashita M, Imai N, Nakazawa R, Sasaki H, et al. Arteriovenous Fistulas after Ultrasound-Guided Needle Biopsy of Kidney Allografts and Treatment Outcomes after Transcatheter Embolization: A Single-center Experience in Japan. Clin Exp Nephrol (2020) 24:963-70. doi:10.1007/s10157-020-01922-8

20. Nicholson ML, Wheatley TJ, Doughman TM, White SA, Morgan JDT, Veitch PS, et al. A Prospective Randomized Trial of Three Different Sizes of CoreCutting Needle for Renal Transplant Biopsy. Kidney Int (2000) 58:390-5. doi:10.1046/j.1523-1755.2000.00177.x

21. Vidhun J, Masciandro J, Varich L, Salvatierra O, Sarwal M Safety and Risk Stratification of Percutaneous Biopsies of Adult-Sized Renal Allografts in Infant and Older Pediatric Recipients. Transplantation (2003) 76:552-7. doi:10.1097/01.tp.0000076097.90123.21

22. Feldmann Y, Böer K, Wolf G, Busch M Complications and Monitoring of Percutaneous Renal Biopsy - a Retrospective Study. Cn (2018) 89:260-8. doi:10.5414/cn109223

23. Patel AG, Kriegshauser JS, Young SW, Dahiya N, Patel MD Detection of Bleeding Complications after Renal Transplant Biopsy. Am J Roentgenology (2021) 216:428-35. doi:10.2214/ajr.20.22990

24. Ferguson C, Winters S, Jackson S, McToal M, Low G. A Retrospective Analysis of Complication and Adequacy Rates of Ultrasound-Guided Native and Transplant Non-focal Renal Biopsies. Abdom Radiol (2018) 43:2183-9. doi:10.1007/s00261-017-1405-z

25. Harrison KL, Nghiem HV, Coldwell DM, Davis CL Renal Dysfunction Due to an Arteriovenous Fistula in a Transplant Recipient. Jasn (1994) 5:1300-6. doi:10.1681/asn.v561300

26. Kolb LG, Velosa JA, Bergstralh EJ, Offord KP Percutaneous Renal Allograft Biopsy. Transplantation (1994) 57:1742-5. doi:10.1097/00007890-199457120-00010

27. Reschen ME, Mazzella A, Sharples E A Retrospective Analysis of the Utility and Safety of Kidney Transplant Biopsies by Nephrology Trainees and Consultants. Ann Med Surg (2018) 28:6-10. doi:10.1016/j.amsu.2018.02.001

28. Plattner BW, Chen P, Cross R, Leavitt MA, Killen PD, Heung M Complications and Adequacy of Transplant Kidney Biopsies: A Comparison of Techniques. J Vasc Access (2018) 19:291-6. doi:10.1177/1129729817747543

Copyright $\odot 2022$ Pirklbauer, Berger, Boban and Tiefenthaler. This is an open-access article distributed under the terms of the Creative Commons Attribution License (CC $B Y$ ). The use, distribution or reproduction in other forums is permitted, provided the original author(s) and the copyright owner(s) are credited and that the original publication in this journal is cited, in accordance with accepted academic practice. No use, distribution or reproduction is permitted which does not comply with these terms. 